\title{
RADICAL PROPERTIES DEFINED LOCALLY BY POLYNOMIAL IDENTITIES II
}

\author{
B. J. GARDNER \\ (Received 3 January: revised 4 July 1978) \\ Communicated by $\mathbf{H}$. Lausch
}

\begin{abstract}
A radical class $\mathscr{R}$ of rings (not necessarily associative) is called an $n$-radical class if it has the property that a ring is in $\mathscr{R}$ if and only if every subring generated by $\leqslant n$ elements is in $\mathscr{R}$. A transfer theorem is proved, relating $n$-radical classes in two universal varieties which share the same $\leqslant n$-generator rings. Partially through the use of this result, we obtain information about extensionclosed subvarieties of various universal varieties of power-associative rings.
\end{abstract}

Subject classification (Amer. Math. Soc. (MOS) 1970): 16 A 21.

In this paper we continue the investigation begun in Part I (Gardner (1979)) of local radical classes of rings, and preserve the notation and terminology of that paper. We begin in Section 1 with a theorem on extension-closed varieties (or, equivalently, direct product-closed local radical classes), showing that such classes can in many instances be dealt with in terms of rings of prime characteristic. It is an open question whether a local radical class which is closed under direct products must be an $n$-radical class for some $n$, and it is not clear what the radical filter would look like if such a class were not an $n$-radical class. However, for $n$-radical classes, closure under direct products turns out to be equivalent to closure of the corresponding $\boldsymbol{n}$-radical filter under arbitrary intersections (Section 2 ). It can happen that for two distinct universal varieties $\mathscr{U}$ and $\mathscr{W}$ the free rings on $\boldsymbol{n}$ generators (for some $n$ ) coincide. This leads to strong connections between $n$-radical classes in the two settings; this matter is discussed in Section 3. In Section 4, the result in Section 3 is applied to extension-closed varieties in power-associative universal varieties; this section also contains more detailed information about extension-closed varieties ( = semi-simple radical classes) of alternative and Jordan rings, the latter 
furnishing examples of extension-closed varieties which are not 1-radical classes. The result of Section 3 is finally used, in Section 5, to extend to alternative rings results obtained by Freidman (1958) for associative rings with nil commutator ideals. All rings considered are power-associative

\section{Extension-closed varieties}

When the universal class $\mathscr{W}$ is a variety, a subvariety of $\mathscr{W}$ is a radical class if and only if it is closed under extensions in $\mathscr{W}$ (see Gardner (1975), Wiegandt (1974)). In this section we prove a theorem which reduces the problem of finding extensionclosed varieties of rings (in many cases) to the corresponding problem for algebras over a finite prime field.

If, for a variety $\mathscr{V}$, there are subvarieties $\mathscr{V}_{1}, \ldots, \mathscr{V}_{n}$ such that every ring in $\mathscr{V}$ is uniquely expressible as a direct product $A_{1} \times \ldots \times A_{n}$, with $A_{i} \in \mathscr{V}_{i}, \mathscr{V}$ is called the product of $\mathscr{V}_{1}, \ldots, \mathscr{V}_{n}$ and for this we use the notation

$$
\mathscr{V}=\mathscr{V}_{1} \times \ldots \times \mathscr{V}_{n} .
$$

As before, $F$ denotes a free $\mathscr{W}$-ring on a countably infinite set of generators. For each $n$, let $F^{n}$, as usual, denote the subring of $F$ generated by all words of length $\geqslant n$.

THEOREM 1.1. Let $\mathscr{W}$ be such that $\bigcap F^{n}=0$, and let $\mathscr{V} \neq\{0\}$ be an extensionclosed variety in $\mathscr{W}$.

(i) If there is a ring $A \in \mathscr{V}$ such that $A^{2}=0 \neq A$, then $\mathscr{V}=\mathscr{W}$.

(ii) If $\mathscr{V} \neq \mathscr{W}$, there are finitely many primes $p_{1}, \ldots, p_{n}$, and finitely many extensionclosed subvarieties $\mathscr{V}_{1}, \ldots, \mathscr{V}_{n}$ of $\mathscr{V}$ such that

$$
\mathscr{r}=\mathscr{r}_{1} \times \ldots \times \mathscr{V}_{n}
$$

and every ring in $\mathscr{V}_{i}$ has characteristic $p_{i}$.

Proof. (i) This is just Corollary 1.9 of Gardner (1975).

(ii) By (i), $\mathscr{V}$ can contain no zerorings. Hence if $0 \neq a \in A \in \mathscr{V}$, we have $\langle a\rangle\langle a\rangle^{2} \in \mathscr{V}$ and so $\langle a\rangle=\langle a\rangle^{2}$ and $a=\sum_{i=2}^{k} n_{i} a^{i}$ for some integers $n_{2}, \ldots, n_{k}$. By Theorem 13.2, p. 321 of Osborn (1972), $A$ is a direct sum of periodic rings of prime characteristic. Suppose infinitely many prime characteristics $p_{1}, p_{2}, \ldots$ occur in this way throughout $\mathscr{V}$. Let $B_{i} \neq 0$ be a ring in $\mathscr{V}$ with characteristic $p_{i}$, for each $i$. Then $\prod B_{i}$ is in $\mathscr{V}$, but it is not a torsion ring-a contradiction. Thus there are only finitely many primes $p_{1}, \ldots, p_{n}$ involved, and using the primary decomposition, we have $\mathscr{V}=\mathscr{V}_{1} \times \ldots \times \mathscr{V}_{n}$, where

$$
\mathscr{V}_{i}=\left\{A \in \mathscr{V} \mid p_{i} A=0\right\}=\left\{A \in \mathscr{V} \mid A \text { has a } p_{i} \text {-primary additive group }\right\},
$$

and so each $\mathscr{V}_{i}$ is both a radical class and a variety. 
Thus in studying extension-closed varieties we can effectively restrict our attention to varieties of rings with prime characteristics. If rings of certain characteristics are in some sense exceptional, as often happens with 2 or 3, we can still obtain partial results by excluding from consideration varieties where the troublesome characteristics are involved and without the necessity of changing from rings to algebras over a ring containig $\frac{1}{2}$ or $\frac{1}{6}$ and so on.

\section{Filters associated with extension-closed varieties}

Since a local radical class is strongly hereditary and homomorphically closed; such a class is closed under direct products if and only if it is an extension-closed variety. We now examine the $n$-radical filters associated with $n$-radical classes which are closed under direct products. The next theorem is analogous to Theorem 2.1 of Jans (1965) dealing with the filter of left ideals associated with a TTF class.

THEOREM 2.1. Let $\mathscr{R}$ be an $n$-radical class with associated $n$-radical filter $\mathscr{F}$. The following conditions are equivalent.

(i) $\mathscr{R}$ is closed under direct products.

(ii) $\mathscr{F}$ is closed under intersections.

Proof. (i) $\Rightarrow$ (ii): Let $\left\{I_{\lambda} \mid \lambda \in \Lambda\right\} \subseteq \mathscr{F}$. Then for each $\lambda \in \Lambda$, there is a ring $A_{\lambda} \in \mathscr{R}$ with elements $a_{1}^{\lambda}, \ldots, a_{n}^{\lambda}$ such that $I_{\lambda}=I_{n}\left(a_{1}^{\lambda}, \ldots, a_{n}^{\lambda}\right)$. Now $\left\langle a_{1}^{\lambda}, \ldots, a_{n}^{\lambda}\right\rangle \in \mathscr{R}$ for each $\lambda$, so $\prod_{\lambda \in \Lambda}\left\langle a_{1}^{\lambda}, \ldots, a_{n}^{\lambda}\right\rangle \in \mathscr{R}$. Let $p=p\left(x_{1}, \ldots, x_{n}\right)$ be in $F$. Then

$$
p\left(\left(a_{1}^{\alpha}\right)_{\Lambda}, \ldots,\left(a_{n}^{\alpha}\right)_{\Lambda}\right)=\left(p\left(a_{1}^{\alpha}, \ldots, a_{n}^{\alpha}\right)\right)_{\Lambda},
$$

so that $p\left(\left(a_{1}^{\lambda}\right)_{\Lambda}, \ldots,\left(a_{n}^{\lambda}\right)_{\Lambda}\right)=0$ if and only if $p\left(a_{1}^{\lambda}, \ldots, a_{n}^{\lambda}\right)=0$ for each $\lambda \in \Lambda$, that is $I_{n}\left(\left(a_{1}^{\lambda}\right)_{\Lambda}, \ldots,\left(a_{n}^{\lambda}\right)_{\Lambda}\right)=\bigcap_{\lambda \in \Lambda} I_{n}\left(a_{1}^{\lambda}, \ldots, a_{n}^{\lambda}\right)=\bigcap_{\lambda \in \Lambda} I_{\lambda}$. But $\left(a_{1}^{\lambda}\right)_{\Lambda}, \ldots,\left(a_{n}^{\lambda}\right)_{\Lambda} \in \prod_{\lambda \in \Lambda} A_{\lambda} \in \mathscr{R}$ and so $\bigcap_{\lambda \in \Lambda} I_{\lambda}$ is in $\mathscr{F}$.

(ii) $\Rightarrow$ (i): Let $\left\{A_{\lambda} \mid \lambda \in \Lambda\right\}$ be a set of rings in $\mathscr{R}$ and let $b_{1}=\left(a_{1}^{\lambda}\right)_{\Lambda}, \ldots, b_{n}=\left(a_{n}^{\lambda}\right)_{\Lambda}$ be in $\prod_{\lambda \in \Lambda} A_{\lambda}$. Then as above,

$$
I_{n}\left(b_{1}, \ldots, b_{n}\right)=\bigcap_{\lambda \in \Lambda} I_{n}\left(a_{1}^{\lambda}, \ldots, a_{n}^{\lambda}\right) \in \mathscr{F},
$$

since, for each $\lambda, a_{1}^{\lambda}, \ldots, a_{n}^{\lambda} \in A_{\lambda} \in \mathscr{R}$. This being so for all $\left(b_{1}, \ldots, b_{n}\right)$, we have $\prod_{\lambda \in \Lambda} A_{\lambda} \in \mathscr{R}$.

If $\mathscr{R}$ is an $n$-radical class which is closed under direct products, then in particular its $n$-radical filter has a smallest member.

THEOREM 2.2. Let $\mathscr{R}$ be an n-radical class which is closcd under direct products and let $\mathscr{F}$ be the associated $n$-radical filter. Then $\mathscr{R}$, as a variety, is defined by $n$-variable identities and the corresponding $T$-ideal of $F_{n}$ is the intersection $J_{0}$ of all the ideals in $\mathscr{F}$. 
Proof. Let $A$ be a ring satisfying all the $n$-variable identities which hold on $\mathscr{R}$. Then every subring $B$ generated by $\leqslant n$ elements is a homomorphic image of the relatively free $\mathscr{R}$-ring on $n$ generators and so $B \in \mathscr{R}$. Since $\mathscr{R}$ is an $n$-radical class, $A$ is in $\mathscr{R}$. Thus $\mathscr{R}$ is defined by $n$-variable identities. Now $J_{0}$ is the set of $n$-variable polynomials vanishing on all rings in $\mathscr{R}$ and so $J_{0}$ is the $n$-variable $T$-ideal associated with $\mathscr{R}$, as asserted.

\section{A transfer theorem}

If $\mathscr{U}$ and $\mathscr{W}$ are two universal varieties containing the same $\leqslant n$-generator rings, for some $n$ (as happens, for instance, when $n=2, \mathscr{U}$ is the variety of associative, $\mathscr{W}$ the variety of alternative, rings) then in particular they have the same $n$-generator free rings, and an $n$-radical filter in one of the universal varieties is an $n$-radical filter in the other. This leads to a connection between $n$-radical classes in the two varieties. We can do a little better than this, however. We have seen examples of $n$-radical classes which can be described in terms of sets $\Phi$ of polynomials: for a ring to belong to such a class, each of its subsets of cardinality $\leqslant n$ must satisfy one or several polynomial identities defined by $\Phi$. It turns out that if the $n$-radical class associated in $\mathscr{U}$ with an $n$-radical filter $\mathscr{F}$ is describable in this way, then the $n$-radical class associated with $\mathscr{F}$ in $\mathscr{W}$ admits exactly the same description. If the set $\Phi$ of polynomials has some suitable composition-closed property, it is of course clear at least that it will define a radical class in both $\mathscr{U}$ and $\mathscr{W}$. It may be, however, that there is no such closure property, but that internal properties of $\mathscr{U}$ (say) must be invoked before we can show that $\Phi$ defines a radical class in $\mathscr{U}$. Use of the transfer theorem which we shall next prove, obviates the necessity for a similar internal argument in $\mathscr{W}$.

The following notation will be useful. Let $\Phi$ be a set of polynomials in $x_{1}, \ldots, x_{n}$, $\mathscr{S}$ a set of subsets of $\Phi$. Let $A$ be a ring such that for every subset $S$ of $A$ with $|S| \leqslant n$, there is a set $\Psi$ in $\mathscr{S}$ such that

$$
p\left(s_{1}, \ldots, s_{n}\right)=0 \text { for all } p \in \Psi \text { and } s_{1}, \ldots, s_{n} \in S .
$$

We denote this situation as follows: $A \sim \mathscr{S}$.

THEOREM 3.1. Let $\mathscr{U}$ and $\mathscr{W}$ be varieties which, for some $n$, have the same free ring $F_{n}$ on $\left\{x_{1}, \ldots, x_{n}\right\}$. Then every $n$-radical filter for $\mathscr{U}$ is an $n$-radical filter for $\mathscr{W}$, and conversely. If, moreover, for some n-radical filter $\mathscr{F}$ there is a set $\Phi$ of polynomials in $F_{n}$ and a set $\mathscr{S}$ of subsets of $\Phi$ such that

$$
\{A \in \mathscr{U} \mid A \sim \mathscr{S}\}
$$

is the n-radical class in $\mathscr{U}$ associated with $\mathscr{F}$, then

$$
\{A \in \mathscr{W} \mid A \sim \mathscr{P}\}
$$

is the $n$-radical class associated with $\mathscr{F}$ in $\mathscr{W}$. 
PrOoF. The first assertion being clear, we proceed to the second. Let $\mathscr{R}, \mathscr{R}$ be the $n$-radical classes associated with $\mathscr{F}$ in $\mathscr{U}, \mathscr{W}$ respectively. If $J \in \mathscr{F}$, then $J=I_{n}\left(a_{1}, \ldots, a_{n}\right)$ for some $a_{1}, \ldots, a_{n} \in A \in \mathscr{R}$, so

$$
F_{n} / J=F_{n} / I_{n}\left(a_{1}, \ldots, a_{n}\right) \cong\left\langle a_{1}, \ldots, a_{n}\right\rangle \in \mathscr{R} .
$$

On the other hand, if $K \triangleleft F_{n}$ and $F_{n} / K \in \mathscr{R}$, then $I_{n}\left(\beta_{1}+K, \ldots, \beta_{n}+K\right) \in \mathscr{F}$ for each $\beta_{1}, \ldots, \beta_{n} \in F_{n}$. Since (by the first defining property of $n$-radical filters) $F_{n} \in \mathscr{F}$, the third defining property for $n$-radical filters implies that $K$ is in $\mathscr{F}$. Thus

$$
\mathscr{F}=\left\{J\left\langle F_{n}\right| F_{n} / J \in \mathscr{R}\right\}=\left\{J\left\langle F_{n}\right| F_{n} / J \in \mathscr{R}^{\prime}\right\} .
$$

Hence

$$
\begin{aligned}
\mathscr{R}^{\prime} & =\left\{A \in \mathscr{W} \mid a_{1}, \ldots, a_{n} \in A \Rightarrow I_{n}\left(a_{1}, \ldots, a_{n}\right) \in \mathscr{F}\right\} \\
& =\left\{A \in \mathscr{W} \mid a_{1}, \ldots, a_{n} \in A \Rightarrow F_{n} / I_{n}\left(a_{1}, \ldots, a_{n}\right) \in \mathscr{R}\right\} \\
& =\left\{A \in \mathscr{W} \mid a_{1}, \ldots, a_{n} \in A \Rightarrow F_{n} / I_{n}\left(a_{1}, \ldots, a_{n}\right) \sim \mathscr{S}\right\} \\
& =\left\{A \in \mathscr{W} \mid a_{1}, \ldots, a_{n} \in A \Rightarrow\left\langle a_{1}, \ldots, a_{n}\right\rangle \sim \mathscr{S}\right\} \\
& =\{A \in \mathscr{W} \mid A \sim \mathscr{S}\} .
\end{aligned}
$$

\section{Applications to 1-radical classes}

We now apply Theorem 3.1 to produce some examples of direct productclosed 1-radical classes in power-associative universal varieties. In the sequel we denote the variety of associative (resp. commutative associative, resp. powerassociative) rings by $\mathscr{A}$ (resp. $\mathscr{C} \mathscr{A}$, resp $\mathscr{P} \mathscr{A}$ ). Note that $\mathscr{C} \mathscr{A}, \mathscr{P} \mathscr{A}$ and any intermediate variety share the same one-generator free rings.

THEOREM 4.1. Let $\mathscr{W}$ be a variety of rings such that $\mathscr{C} \mathscr{A} \subseteq \mathscr{W} \subseteq \mathscr{P} \mathscr{A}$. The following conditions are equivalent for a proper subclass $\mathscr{V} \neq\{0\}$ of $\mathscr{W}$.

(i) $\mathscr{V}$ is a subvariety of $\mathscr{W}$ which is closed under extensions (in $\mathscr{W}$ ) and is defined by one-variable identities.

(ii) $\mathscr{V}$ is a direct product-closed 1-radical class in $\mathscr{W}$.

(iii) There exists a finite set $P$ of primes, and for each $p \in P$ there exists a finite set $N(p)$ of integers $>1$, such that $\mathscr{V}$ is the subvariety of $\mathscr{W}$ defined by the identities

$$
\prod\{p \mid p \in P\} x=0 ; \quad \hat{p} x \prod\left\{x^{p^{n-1}}-1 \mid n \in N(p)\right\}=0 \quad \text { for each } p \in P, \ldots,
$$

where $\hat{p}=\prod\{q|q \in P|\{p\}\}$, or 1 , if $P=\{p\}$.

Proof. The equivalence of (i) and (ii) follows from Theorem 1.4 of Gardner (1975) and the closure properties of local (and in particular 1-) radical classes. 
(ii) $\Rightarrow$ (iii): Let $\mathscr{F}$ be the 1-radical filter associated with $\mathscr{V}$. By Theorem 2.1, there exists an ideal $J_{0}$ of $F_{1}$ such that

$$
\mathscr{F}=\left\{J \mid J_{0} \subseteq J \triangleleft F_{1}\right\}
$$

Let $\mathscr{V}^{\prime}$ denote the 1-radical class defined by $\mathscr{F}$ in $\mathscr{A}$. By Theorem $2.1, \mathscr{V}^{\prime}$ is closed under direct products, so then by results of Gardner and Stewart (1975), $\mathscr{V}^{\prime}$ is the variety (in $\mathscr{A}$ ) defined by a set of identities of the form $\left({ }^{*}\right)$. But then Theorem 3.1 implies that $\mathscr{V}$ is defined in $\mathscr{W}$ by this set of identities.

(iii) $\Rightarrow$ (ii): Let $\mathscr{V}$ be the variety defined in $\mathscr{W}$ by a set of identities of the form ${ }^{*}$ ), and let $\mathscr{V}^{\prime}$ be the variety defined in $\mathscr{A}$ by these identities. Then by results of Gardner and Stewart (1975), $\mathscr{V}^{\prime}$ is a radical class, and thus a direct productclosed 1-radical class in $\mathscr{A}$. Let $\mathscr{F}$ be its 1-radical filter, and let $\mathscr{R}$ be the 1-radical class associated in $\mathscr{W}$ with $\mathscr{F}$. By Theorem 3.1,

$$
\mathscr{R}=\left\{A \in \mathscr{W} \mid a \in A \Rightarrow a \text { satisfies }\left(^{*}\right)\right\}=\mathscr{V} .
$$

THEOREM 4.2. Let $\mathscr{W}$ be a variety with $\mathscr{A} \subseteq \mathscr{W} \subseteq \mathscr{P} \mathscr{A}$. Then for every non-trivial extension-closed variety $\mathscr{U}$ in $\mathscr{W}$, either there exists a set of identities of the form $\left(^{*}\right)$ defining a variety $\mathscr{V}$ such that $\mathscr{U} \subseteq \mathscr{V}$, or $\mathscr{A} \subseteq \mathscr{U}$.

PRoOF. If $\mathscr{U}$ contains a ring $A$ such that $A^{2}=0 \neq A$, then $A \in \mathscr{A} \cap \mathscr{U}$, so by Corollary 1.9 of Gardner (1975), $\mathscr{U} \cap \mathscr{A}=\mathscr{A}$. If, on the other hand, there is no such $A$ in $\mathscr{U}$, then for $a \in R \in \mathscr{U}$ we have $\langle a\rangle\langle a\rangle^{2} \in \mathscr{U}$, so that $a \in\langle a\rangle^{2}$ and thus every ring in $\mathscr{U}$ is periodic (see Osborn (1972), pp. 318-322). Now $\langle a\rangle$ is associative (and periodic) for each $a \in R \in \mathscr{U}$ and so (if $a \neq 0$ ) is a direct sum of a finite number of finite fieids (Stewart (1970), Theorem 3.4). Let $\mathscr{K}$ be the set of fields occurring in this way as $R$ varies over $\mathscr{U}$. Clearly $\mathscr{K}$ is strongly hereditary. Arguing as in the proof of Theorem 4.3 of Stewart (1970), one can show that $\mathscr{K}$ is finite. Let $P$ be the set of characteristics of fields in $\mathscr{K}$, and for each $p \in P$, let

$$
N(p)=\left\{n \mid \mathscr{K} \text { contains the field of order } p^{n}\right\} .
$$

This choice of $P$ and the $N(p)$ determines a set of identities of the form ( $\left.{ }^{*}\right)$ and it is clear that $\mathscr{U}$ is contained in the corresponding variety.

It is not known whether the second possibility mentioned in the theorem can actually occur.

All the examples of extension-closed varieties thus far exhibited have been 1-radical classes. We shall shortly see that there are extension-closed varieties which are not of this type. This is an appropriate point to mention a result of Loustau (1971): in $\mathscr{P}_{\mathscr{A}}$, the variety defined by the identities $p x=0, x^{p}=x$, where $p$ is an odd prime, consists of associative rings and is thus a minimal variety (being generated by GF $(p)$ ). (Note that this set of identities has the form $\left({ }^{*}\right)$.) 
Thus to get non-1-radical examples of extension-closed varieties involving such characteristics $p$, one must let in more fields. For characteristic 2, Loustau's result ceases to hold, as can be seen from the non-associative division rings satisfying $x^{2}=x$, presented by Fiedorowicz (1974).

For alternative and Jordan rings we can obtain some more precise information about extension-closed varieties.

THEOREM 4.3. For a non-trivial variety $\mathscr{V}$ of alternative rings, the following conditions are equivalent.

(i) $\mathscr{V}$ is a semi-simple radical class;

(ii) $\mathscr{V}$ has attainable identities;

(iii) $\mathscr{V}$ is extension-closed;

(iv) $\mathscr{V}$ is defined by a set of identities of the form $\left({ }^{*}\right)$.

Proof. The equivalence of (i), (ii) and (iii) follows from Theorem 3.3 (proof) and Corollary 1.12 of Gardner (1975). By Theorem 4.1, we need only prove (iii) $\Rightarrow$ (iv). By Theorem 1.1, $\mathscr{V}$ consists of periodic rings; in particular, no ring in $\mathscr{V}$ has nilpotent elements. By Proposition 3.1 of Ryabukhin (1969a) and Theorem 1.1 of Ryabukhin (1969b) (see also Hentzel (1974)), each ring in $\mathscr{V}$ is a subdirect product of rings without zero divisors. Let $R \neq 0$ be an alternative periodic ring without zero divisors. If $0 \neq a \in R$, then $a=a^{n}$ for some $n>1$. If $\mathrm{b}$ is any non-zero element of $R$, we have $a\left(a^{n-1} b\right)=\left(a a^{n-1}\right) b=a^{n} b=a b$. Since $a$ is not a zero divisor, we have $a^{n-1} b=b$. Similarly, $b a^{n-1}=b$ and it follows that $a^{n-1}$ is an identity fod $R$. Then (compare the proof of Lemma 3.2 of Stewart (1970)) $a^{n-1} \in\langle a\rangle=\langle a\rangle^{2}$ and so $a^{n-1} \in\langle a\rangle a \subseteq R a$ and $a^{n-1} \in a\langle a\rangle \subseteq a R$, which means that $R$ is a division ring. The Cayley-Dickson/algebras are not periodic and so we conclude that every alternative periodic ring is a subdirect product of periodic fields. Thus, $\boldsymbol{V}$ is generated (as a variety) by fields, and familiar arguments complete the proof.

For Jordan rings the characteristic 2 case presents difficulties, so here we will discuss only varieties of rings with odd prime characteristic. Theorem 1.1 tells how to put these together to form more general varieties but, at the same time, to avoid the troublesome characteristic 2 .

THEOREM 4.4. The following conditions are equivalent for a variety $\mathscr{V}$ of Jordan rings such that

$$
\{0\} \nsubseteq \mathscr{V} \subseteq\{A \mid p A=0\}
$$

for an odd prime $p$.

(i) $\mathscr{V}$ is a semi-simple radical class;

(ii) $\mathscr{V}$ has attainable identities;

(iii) $\mathscr{V}$ is extension-closed; 
(iv) there is a finite set $N(p)$ of integers $>1$ such that $\mathscr{V}$ is contained in the variety defined by the identities

$$
p x=0 ; \quad x \prod\left\{x^{p^{n}-1}-1 \mid n \in N(p)\right\}=0 .
$$

Proof. (i) and (ii) are equivalent, as noted above. Also (ii) implies (iii) (see Mal'tsev (1967) or Gardner (1975), Corollary 1.6).

(iii) $\Rightarrow$ (iv): This is a special case of Theorem 4.2 .

(iv) $\Rightarrow$ (ii): If $a \in A \in \mathscr{V}$, then $\langle a\rangle \in \mathscr{V}$ and $\langle a\rangle$ is associative. Since it satisfies the stated identities, $a$ is a finite direct sum of finite fields and thus periodic. Hence $A$ is periodic and so $\mathscr{V}$ consists of periodic rings. By Theorem 15.11, p. 354, of Osborn (1972) each ring in $\mathscr{V}$ is a subdirect product of simple rings and $\mathscr{V}$ is then the variety generated by the class $\mathscr{G}$ of simple rings appearing in this way, that is the class of simple rings in $\mathscr{V}$. Now by the same theorem of Osborn, a simple, periodic Jordan ring is either a field or a ring of the form

$$
H=\left\{\left[\begin{array}{ll}
a & b u \\
b & c
\end{array}\right] \quad a, b, c \in K\right\}
$$

where $K$ is a periodic field, $-u$ is not a square and the multiplication is the usual Jordan product on the ring $\left[\begin{array}{ll}K & K \\ K & K\end{array}\right]$. By the argument quoted in the proof of Theorem 4.2, $\mathscr{V}$ can only contain finitely many fields and all of them are finite. Since the ring $H$ described above has $K$ as a subring, $K$ must be in $\mathscr{V}$ if $H$ is and so $K$ must then be finite. Since there are only finitely many fields in $\mathscr{V}$, it follows that there must be only finitely many simple rings in $\mathscr{V}$ and that each of these is finite. An application of Theorem 3.3 of Gardner (1975) completes the proof.

COROLlaRY 4.5. Let $p$ be an odd prime, $N(p)$ a finite set of integers $>1$. The following set of identities is attainable for Jordan rings:

$$
\left\{p x=0, x \prod\left\{x^{p^{n}-1}-1 \mid n \in N(p)\right\}=0,(x y) z-x(y z)=0\right\} .
$$

Proof. Let $\mathscr{V}$ be the variety defined by the first two identities; the fields in $\mathscr{V}$ generate the variety defined by all three.

COROLLARY 4.6. In the universal variety of Jordan rings there are 3-radical classes which are closed under direct products and which are not 1-radical classes.

\section{An application to 2-radical classes}

Let $\mathscr{N}$ denote the class of nil associative rings, $\mathscr{C}$ the class of commutative associative rings. As noted in Section 4 of Part I, the class

$$
\mathscr{N} \circ \mathscr{C}=\{A \mid A \text { has an ideal } I \in \mathscr{N} \text { with } A / I \in \mathscr{C}\},
$$


or, equivalently, the class of rings with nilpotent commutators in which the set of nilpotent elements is an ideal, is a 2 -radical class. To avoid confusion, in the sequel we shall denote by $\overline{\mathcal{N}}$ (resp. $\overline{\mathscr{C}}$ ) the class of nil (resp. commutative) alternative rings.

THEOREM 5.1. $\overline{\mathscr{N}} \mathrm{OC}$ is a 2-radical class.

Proof. The free alternative ring on $\left\{x_{1}, x_{2}\right\}$ is associative. Let $\mathscr{F}$ be the 2 -radical filter associated with $\mathcal{N} \circ \mathscr{C}$, and let $\mathscr{R}$ be the 2-radical class of alternative rings defined by $\mathscr{F}$.

If $a, b \in A \in \mathscr{R}$, then $\langle a, b\rangle \in \mathscr{R}$ and $I_{2}(u, v) \in \mathscr{F}$ for each $u, v \in\langle a, b\rangle$. Since $\langle a, b\rangle$ is associative, this means that $\langle a, b\rangle \in \mathscr{N} \circ \mathscr{C}$. Thus if $a$ is nilpotent, $a b$ and $b a$ (being in $\langle a, b\rangle)$ are nilpotent, while if $a$ and $b$ are both nilpotent and so is $a-b$. Finally, $a b-b a(\in\langle a, b\rangle \in \mathcal{N} \circ \mathscr{C})$ is nilpotent for any $a, b$. Thus the set of nilpotent elements of $A$ is an ideal (necessarily $\overline{\mathscr{N}}(A)$ ) containing all commutators and so $A \in \bar{N}$ o $\overline{\mathscr{C}}$.

Conversely, if $A \in \overline{\mathcal{N}} \circ \overline{\mathscr{C}}$, the nilpotent elements of $A$ form an ideal containing all commutators (same proof as for associative rings). The same is true of $\langle a, b\rangle$ for every $a, b \in A$. But $\langle a, b\rangle$ is associative and so $\langle a, b\rangle \in \mathscr{N} \circ \mathscr{C} \subseteq \mathscr{R}$. Since $\mathscr{R}$ is a 2-radical class, $A$ is in $\mathscr{R}$. We conclude that $\mathscr{R}=\overline{\mathscr{N}}$ o $\overline{\mathscr{C}}$.

$\bar{N} \circ \overline{\mathscr{C}}$ is, of course, not a 1-radical class.

COROLlaRy 5.2. Let $A$ be an $\overline{\mathcal{N}}$-semi-simple alternative ring of which every nonzero homomorphic image has a non-zero commutative ideal. Then $A$ is commutative.

Proof. Certainly $A$ is in the radical class $\bar{N} \circ \overline{\mathscr{C}}$. But $\overline{\mathscr{N}}(A)=0$ and so $A \in \overline{\mathscr{C}}$.

NoTE. This result can also be obtained from a combination of Theorem $\mathbf{A}^{\prime}$, Section 4 of Slater (1972) and the results of Freidman (1958) which establish the fact that $\mathscr{N} \circ \mathscr{C}$ is a radical class of associative rings. Also, for associative rings, Osborn (1972), Theorem 11.15 has obtained a more general result, using arbitrary standard identities in place of commutativity.

COROLlaRY 5.3. Let $A$ be an alternative ring such that there is a series

$$
0=I_{0} \triangleleft I_{1} \triangleleft I_{2} \triangleleft \ldots \triangleleft I_{\alpha} \triangleleft I_{\alpha+1} \triangleleft \ldots \triangleleft I_{\mu}=A,
$$

where $I_{\beta}=\bigcup_{\alpha<\beta} I_{\alpha}$ if $\beta$ is a limit ordinal, and such that $I_{\alpha+1} / I_{\alpha}$ is in $\bar{N} \cup \overline{\mathscr{C}}$ for all $\alpha$. Then $A \in \overline{\mathcal{N}} \circ \overline{\mathscr{C}}$.

Proof. $I_{0}$ is in $\bar{N} \cup \overline{\mathscr{C}} \subseteq \overline{\mathscr{N}} \circ \overline{\mathscr{C}}$. If $I_{\alpha} \in \bar{N} \circ \overline{\mathscr{C}}$, then from the exact sequence

$$
0 \rightarrow I_{\alpha} \rightarrow I_{\alpha+1} \rightarrow I_{\alpha+1} / I_{\alpha} \rightarrow 0,
$$

with $I_{\alpha} \in \bar{N} \circ \overline{\mathscr{C}}$ and $I_{\alpha+1} / I_{\alpha} \in \overline{\mathscr{N}} \cup \overline{\mathscr{C}} \subseteq \overline{\mathscr{N}}$ $\overline{\mathscr{C}}$, we see that $I_{\alpha+1}$ is in $\bar{N} \circ \overline{\mathscr{C}}$. If $I_{\alpha} \in \overline{\mathscr{N}} \circ \overline{\mathscr{C}}$ 
for all $\alpha<$ a limit $\beta$, then $I_{\beta} \in \bar{N} \circ \overline{\mathscr{C}}$, since the latter is a local radical class. Hence each $I_{\alpha}$ is in $\overline{\mathscr{N}} \circ \overline{\mathscr{C}}$ and so $A=I_{\mu} \in \overline{\mathcal{N}} \circ \overline{\mathscr{C}}$.

COROLlaRY 5.4. If an alternative ring $A$ has a commutative ideal $J$ such that $A / J$ is nil, then $A$ is in $\bar{N} \circ \overline{\mathscr{C}}$ (that is $\mathscr{C} \circ \overline{\mathcal{N}} \subseteq \bar{N} \circ \overline{\mathscr{C}}$ ).

\section{References}

Z. Fiedorowicz (1974), 'The structure of autodistributive algebras', J. Algebra 31, 427-436.

P. A. Freidman (1958), 'On the theory of the radical of an associative ring', Isv. Vyshch. Ucheb. Zaved. Mat. No. 3(4), 225-232 (in Russian).

B. J. Gardner (1979), 'Radical properties defined locally by polynomial identities, I', J. Austral. Math. Soc. (Ser. A) 27, 257-273.

B. J. Gardner (1975), 'Semi-simple radical classes of algebras and attainability of identities', Pacific J. Math. 61, 401-416.

B. J. Gardner and P. N. Stewart (1975), 'On semi-simple radical classes', Bull. Austral. Math. Soc. 13, 349-353.

I. R. Hentzel (1974), 'Alternative rings without nilpotent elements', Proc. Amer. Math. Soc. 42, 373-376.

J. P. Jans (1965), 'Some aspects of torsion', Pacific J. Math. 15, 1249-1259.

J. A. Loustau (1971), 'On a class of power-associative periodic rings', Bull. Austral. Math. Soc. 5, 357-362.

A. I. Mal'tsev (1967), 'Multiplication of classes of algebraic systems', Siberian Math. J. 8, 254-267.

J. M. Osborn (1972), 'Varieties of algebras', Advances in Math. 8, 163-369.

Yu. M. Ryabukhin (1969a), 'Algebras without nilpotent elements, I', Algebra and Logic 8, 103-122.

Yu. M. Ryabukhin (1969b), 'Algebras without nilpotent elements, II', Algebra and Logic 8, 123137.

M. Slater (1972), 'Prime alternative rings, III', J. Algebra 21, 394-409.

P. N. Stewart (1970), 'Semi-simple radical classes', Pacific J. Math. 32, 249-254.

R. Wiegandt (1974), Radical and semisimple classes of rings (Queen's Papers in Pure and Applied Mathematics, No. 37, Kingston, Ontario).

Dalhousie University

Halifax, N.S.

Canada

Current address:

University of Tasmania

Hobart

Australia 